\title{
Estudio de la concentración óptima de fósforo de la estruvita como fertilizante en suelos con mineralogía contrastada
}

\author{
Study of the struvite optimal phosphorus concentration as fertilizer in soils with contrasting minerology
}

\author{
Leticia Angélica Flores Pérez ${ }^{* 1}$, Roberto García Ruíz², Víctor Aranda Sanjuán², Julio A. Calero González² \\ Benemérita Universidad Autónoma de Puebla, Facultad de Ingeniería Química, Ciudad Universitaria, Av. Sn. Claudio y 18 \\ sur, Col. Jardines de San Manuel, 72570 Puebla, Pue., México. \\ Universidad de Jaén. Departamento de Biología Animal, Vegetal y Ecología, Campus Las Lagunillas S/N, 23071, Jaén, Jaén, \\ España.
}

\section{RESUMEN}

La aplicación de la estruvita y otros fosfatos procedentes del reciclado de residuos de diversa índole con potencial fertilizante es ya una realidad. Sin embargo, no se conoce aún en profundidad su valor real como fertilizante en suelos con mineralogía contrastada y la concentración óptima en el desarrollo de las plantas. En este trabajo se determinó la concentración óptima del fertilizante para el desarrollo adecuado de Lepidium sativum como planta modelo en suelos de propiedades contrastantes; olivares sobre margas $(\mathrm{pH} 8.14)$ y granodioritas ( $\mathrm{pH}$ 6.74), con concentraciones crecientes de estruvita y superfosfato $\left(0\right.$ a $\left.32 \mu \mathrm{g} \mathrm{P} \mathrm{mL}^{-1}\right)$. Los resultados obtenidos indican que las concentraciones óptimas para que la planta alcance su desarrollo óptimo está entre 0 a 6 ppm en suelos ácidos con estruvita, mientras que en suelos básicos se presenta un efecto inhibitorio en el desarrollo, así mismo a mayores concentraciones de fertilizante las plantas disminuyen su supervivencia con los dos fertilizantes estudiados.

Palabras clave: estruvita, superfosfato, granodiorita, marga, supervivencia.

\section{ABSTRACT}

The application of struvite and other phosphates from the recycling of waste from different sources with fertilizer potential is now a reality. However, the use as fertilizer in soils with different properties and the optimum concentration for the growth of plants is unknown. The aim of this study was to assess of optimum fertilizer concentration for the best growth of Lepidium sativum as model plant in soils of contrasting properties; calcareous soil on marl ( $\mathrm{pH} \mathrm{8.14)}$ and siliceous soil on granodiorites ( $\mathrm{pH}$ 6.74), with increasing concentrations of P-struvite and P-superphosphate (0 to $32 \mu \mathrm{g}$ $\left.\mathrm{P} \mathrm{mL}^{-1}\right)$. The results indicate that optimal growth- promoting concentrations for the plant are between 0 to $6 \mathrm{ppm}$ in acidic soils with struvite, while in basic soils there is a growth inhibitory effect, likewise at higher fertilizer concentrations plants survival decrease with the two types of fertilizer assayed.

Key words: struvite, superphosphate, granodiorite, marl, survival.

\section{INTRODUCCIÓN}

El fósforo $(P)$ y el nitrógeno son los nutrientes esenciales para el adecuado desarrollo de las plantas, no obstante, las deficiencias de uno de estos nutrientes limitan la productividad agrícola. Se sabe que la principal fuente de $\mathrm{P}$ en los sistemas agrícolas es el superfosfato y según estudios realizados por diversas fuentes institucionales estiman que el $90 \%$ de $P$ que se extrae se destina a la agricultura como fertilizante (Kataki et al., 2016), esto ha puesto en alarma a las entidades gubernamentales y no gubernamentales por el agotamiento de las reservas naturales de $P$ en los próximos 100 años cuya fuente no es renovable (Wang et al., 2016), poniendo en riesgo la sostenibilidad de la productividad agroalimentaria y la seguridad alimentaria (Huijun et al., 2016).

Debido a la gran necesidad y los problemas relacionados con la crisis de $\mathrm{P}$ en las fuentes de reserva natural, han surgido diversas investigaciones que sugieren una serie de alternativas para optimizar el uso de fertilizantes de $\mathrm{P}$ y el desarrollo de nuevas tecnologías integrales para la obtención de P por medio de procesos de reciclado de residuos orgánicos procedentes de desechos urbanos, industriales y agrícolas. Algunas experiencias indican que el estiércol representa entre el 21 a 90\% de P total (Wang et al., 2016). Otros estudios apuntan que el reciclaje de $P$ de la orina y de las heces supondría el suministro del $22 \%$ de la demanda de $\mathrm{P}$ a nivel mundial (Metson et al., 2016).

Actualmente la estruvita o MAP (Fosfato de magnesio y amonio hexahidratado- $\mathrm{MgNH}_{4} \mathrm{PO}_{4} \cdot 6 \mathrm{H}_{2} \mathrm{O}$ ) es considerada como una fuente alternativa de sustitución de fertilizantes químicos, por su viabilidad económica y técnica para su obtención sin involucrar ningún impacto ambiental, ya que la principal fuente de obtención, de acuerdo a las investigaciones recabadas procede de los desechos de origen urbano, industrial y agrícola (Metson et al., 2016; Wang et al., 2016), la cual contiene una cantidad considerable de $\mathrm{P}$ (aguas residuales del curtido de cuero 2.5 a $8 \mathrm{mg} \mathrm{P}^{\mathrm{P}}$ total $\mathrm{L}^{-1}$, efluente del envasado de productos cárnicos 5.5 a $10 \mathrm{mg} \mathrm{P}$ total $\mathrm{L}^{-1}$,

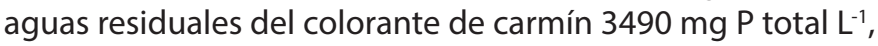

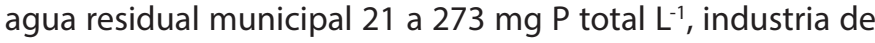
la levadura $17.4 \mathrm{mg} P$ total $\mathrm{L}^{-1}$, orina humana 240 a $460 \mathrm{mg}$ $P$ total $L^{-1} y$ en general en la industria láctea representa un 
contenido de $9.3 \mathrm{~g} \mathrm{P}$ total $\mathrm{kg}^{-1}$, granja de pollos $18 \mathrm{~g} \mathrm{P}$ total

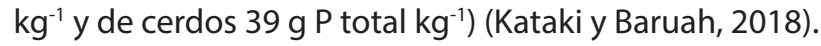

Los estudios abordados a escala piloto de la estruvita como fertilizante en invernaderos indican que la disponibilidad de $P$ en el suelo añadiendo estruvita como fertilizante dependía en gran medida de las propiedades físico-químicas del suelo, la presencia de microorganismos y de la liberación de compuestos orgánicos que condicionaran la disolución/ adsorción/fijación de P en la planta (Maaß et al., 2014; Talboys et al., 2016; Vaneeckhaute et al., 2016).

Si bien la recuperación y aplicación de estruvita y otros fosfatos con potencial fertilizante es ya una realidad (Johnston y Richards, 2003; González y García, 2007; Plaza et al., 2007; Pastor et al., 2008; Massey et al., 2009; Cabeza et al., 2011), no se conoce aún en profundidad su valor real como fertilizante en cultivos concretos, como es el caso del olivar, y sobretodo hasta qué punto o concentración de estruvita como fuente de $\mathrm{P}$ puede ser tóxico para el desarrollo de la planta, en especial en suelos de diferentes propiedades.

El objetivo del presente trabajo fue determinar la concentración óptima de fertilizante para el desarrollo del tallo y de la raíz de una planta modelo (Lepidium sativum) a diferentes concentraciones de estruvita en suelos con mineralogía contrastada; suelo silíceo sobre granodiorita y suelo carbonatado sobre margas.

\section{MATERIALES Y MÉTODOS}

Se tomaron muestras de la capa arable (primeros 30 $\mathrm{cm}$ ) de los dos tipos de suelo (sobre margas a pH básico y sobre granito a $\mathrm{pH}$ ácido) en olivares comerciales de la provincia de Jaén ( $37^{\circ} 49^{\prime} 58.25^{\prime \prime} \mathrm{N}$ y $\left.3^{\circ} 46^{\prime} 06.05^{\prime \prime} \mathrm{O}\right)$ y Córdoba ( $38^{\circ} 09^{\prime} 01.10^{\prime \prime} \mathrm{N}$ y $4^{\circ} 54^{\prime} 17^{\prime \prime} \mathrm{O}$ ). Las muestras de suelo se ta- mizaron $(<2 \mathrm{~mm})$ y las principales propiedades se presentan en la Tabla 1.

Se desarrollaron dos ensayos comparativos en ausencia y en presencia de suelo, en la que se prepararon soluciones de concentraciones $0,3,6,10,17$ y $31 \mu \mathrm{g} \mathrm{P} \mathrm{mL}^{-1}$ en forma de estruvita (Struvite Crystal Green ${ }^{\circledast}$ de Ostara Nutrient Recovery Technologies Inc., Tabla 2) y de superfosfato (SS) (FERTYSEM Agro Special SL, Tabla 2) que se dispusieron en tubos falcón de $50 \mathrm{~mL}$. En los ensayos con ausencia de suelo sólo se agregó agua destilada, mientras que en los ensayos en presencia de suelo se agregaron $3 \mathrm{~g}$ de suelo (suelos silíceos sobre granito y/o suelos carbonatados sobre marga). Las disoluciones se agitaron a una velocidad de $42 \mathrm{U} \mathrm{min}^{-1}$ durante $72 \mathrm{~h}$ a temperatura ambiente. Posteriormente las disoluciones se emplearon para la germinación de semillas, el crecimiento radicular y del tallo de la planta modelo $L$. sativum.

Los experimentos de germinación se desarrollaron de acuerdo al test de Zucconi et al. (1981) con modificaciones en el volumen, tipo de medio de cultivo y del fotoperiodo (Mbarka et al., 2010; Ofosu-Budu et al., 2010; Boluda et al., 2011; El Fels et al., 2014; Khan et al., 2014).

Se dispusieron 10 semillas de $L$. sativum ROCALBA ${ }^{\oplus}$ sobre papel filtro en cajas petri estériles de $9 \times 1.5 \mathrm{~cm}$ y papel filtro $A B E T^{\circledast} 145$ de $9 \mathrm{~cm}$ de diámetro. Se añadieron $5 \mathrm{~mL}$ de la disolución preparada y se incubaron durante 10 días en una incubadora (CLIMAS LAB) con un fotoperiodo de $12 \mathrm{~h} \mathrm{L/O} \mathrm{a}$ $27^{\circ} \mathrm{C}$. Se monitorizó la germinación, el desarrollo del tallo y de la raíz cada 24 horas. Tras el periodo de incubación se contabilizaron las semillas germinadas y se midió la longitud radicular y del tallo de las plántulas con una regla graduada. Todos los datos obtenidos de los ensayos se utilizaron para

Tabla 1. Propiedades del suelo silíceo sobre granodiorita y del suelo carbonatado sobre marga.

Table 1. Properties of siliceous soil on granodiorite y carbonate soil on marl.

\begin{tabular}{|c|c|c|c|c|c|c|c|c|c|c|}
\hline \multirow{2}{*}{ Muestra } & \multicolumn{4}{|c|}{ Clasificación textural } & \multicolumn{6}{|c|}{ Propiedades físicas } \\
\hline & Arena (\%) & Limo (\%) & Arcilla (\%) & $\begin{array}{l}\text { Clasifica- } \\
\text { ción USDA }\end{array}$ & $\begin{array}{l}\text { D.A. (g/ } \\
\text { Cc) }\end{array}$ & C.C. (\%) & P.M. (\%) & Agua útil (\%) & S.A. (\%) & $\begin{array}{l}\text { Permeabili- } \\
\text { dad ( } \mathrm{mm} / \mathrm{h})\end{array}$ \\
\hline Granito & 63 & 23 & 13 & Fr-Arenoso & 1.55 & 18.4 & 9.0 & 9.4 & 41.6 & 31.6 \\
\hline Marga & 28 & 48 & 23 & Franco & 1.43 & 31.2 & 15.3 & 15.9 & 46.2 & 9.3 \\
\hline
\end{tabular}

Propiedades químicas

\begin{tabular}{|c|c|c|c|c|c|c|c|c|c|c|c|c|c|c|c|c|c|c|c|c|c|c|c|}
\hline & \multirow{2}{*}{$\mathbf{N} \%$} & \multicolumn{4}{|c|}{$\begin{array}{c}\text { Bases de cambio } \\
(\mathbf{m g} / \mathbf{K g})\end{array}$} & \multirow[b]{2}{*}{$\mathrm{Na}$} & \multirow{2}{*}{ C.I.C. ${ }^{2}$} & \multirow{2}{*}{$\mathbf{A l}^{2}$} & \multicolumn{5}{|c|}{ \% de saturación } & \multicolumn{2}{|c|}{$\mathrm{pH}$} & \multirow{2}{*}{ C.E. ${ }^{3}$} & \multirow{2}{*}{$\begin{array}{c}\text { M.O. } \\
\%\end{array}$} & \multirow{2}{*}{$\mathrm{C} / \mathrm{N}$} & \multirow{2}{*}{$\begin{array}{c}\text { Caliza } \\
\text { activa } \\
\%\end{array}$} & \multirow{2}{*}{$\begin{array}{c}(\mathrm{Ca}+\mathrm{Mg}) \\
\mathrm{K}\end{array}$} & \multirow{2}{*}{$\mathrm{Ca} / \mathrm{K}$} & \multirow{2}{*}{$\begin{array}{l}\mathrm{Ca} / \\
\mathrm{Mg}\end{array}$} & \multirow{2}{*}{$\mathrm{Mg} / \mathrm{K}$} \\
\hline & & $\mathbf{P}^{1}$ & $\mathbf{K}$ & $\mathrm{Ca}$ & Mg & & & & $\mathbf{K}$ & Mg & $\mathrm{Ca}$ & $\mathrm{Na}$ & Bases & $\mathrm{H}_{2} \mathrm{O}$ & $\mathrm{KCl}$ & & & & & & & & \\
\hline Granito & 0.05 & 7 & 43 & 548 & 109 & 36 & 10 & 6 & 1 & 9 & 27 & 2 & 39 & 6.74 & 6.05 & 0.184 & 1.35 & 17.2 & $<1$ & 33 & 25 & 3 & 8 \\
\hline Marga & 0.08 & 31 & 348 & 2744 & 156 & 64 & 19 & 3 & 5 & 7 & 72 & 1 & 85 & 8.14 & 7.44 & 0.219 & 2.05 & 14.7 & 75 & 17 & 15 & 11 & 1 \\
\hline
\end{tabular}

1: Fósforo Olsen; 2: Unidades meq/100g; 3: Relación 1:5 a $25^{\circ} \mathrm{C}$ (mS/cm); DA: Densidad aparente; C.C. Capacidad de campo; P.M.: Punto de marchitamiento; S.A.: Grado de saturación en agua; Al: Acidez Intercambiable en meq/100g. C.I.C.: Capacidad de Intercambio Catiónico; C.E.: Conductividad Eléctrica; M.O.: Materia Orgánica; C/N: Relación Carbono/Nitrógeno

1: Olsen Phosphorus; 2: Units meq/100g; 3: Relation 1:5 to $25^{\circ} \mathrm{C}$ (mS/cm); DA: Density apparent; C.C. Field Capacity; W.P.: Wilting Point; A.W.: Available Water; W.S.: Water Saturation; IA: Interchange Acid in meq/100g; C.E.C.: Cation Exchange Capacity; E.C.: Electrical Conductivity; O.M.: Organic Matter; C/N: Relationship between Carbon/Nitrogen 
Tabla 2. Principales propiedades de la estruvita y superfosfato (SS). Table 2. Main properties of struvite and superphosphate (SS).

\begin{tabular}{|c|c|c|c|}
\hline Estruvita/Nutriente & $\begin{array}{c}\text { Contenido } \\
(\%)\end{array}$ & SS/Nutriente & $\begin{array}{c}\text { Contenido } \\
(\%)\end{array}$ \\
\hline N amoniacal & 5 & $\begin{array}{c}\mathrm{P}_{2} \mathrm{O}_{5} \text { soluble en } \\
\text { citrato amónico } \\
\text { neutro }\end{array}$ & 18 \\
\hline $\begin{array}{l}\mathrm{P}_{2} \mathrm{O}_{5} \text { total } / \mathrm{P}_{2} \mathrm{O}_{5} \text { dispo- } \\
\text { nible }\end{array}$ & 28 & $\begin{array}{c}\mathrm{P}_{2} \mathrm{O}_{5} \text { soluble en } \\
\text { agua }\end{array}$ & 12 \\
\hline $\mathrm{Mg}$ & 10 & $\mathrm{SO}_{3}$ & 28 \\
\hline
\end{tabular}

determinar el porcentaje de índice de germinación (PIG), longitud de la raíz (PLR) y la longitud del tallo (PLT).

$$
\begin{aligned}
& \text { Número de semillas Longitud promedio de } \\
& \mathrm{PIG}=\frac{\text { germinadas en el extracto }}{\text { Número de semillas }} \times \frac{\text { las raíces en el extracto }}{\text { Longitud promedio de }} \times 100 \\
& \text { germinadas en el control las raíces en el control } \\
& \text { PLR }=\frac{\begin{array}{l}
\text { Longitud promedio de las } \\
\text { raíces en el extracto }
\end{array}}{\text { Longitud promedio de las }} \times 100 \\
& \text { raíces en el control }
\end{aligned}
$$

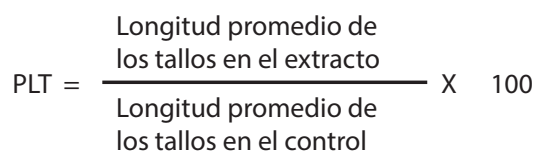

Los resultados se trataron estadísticamente empleando un análisis estadístico descriptivo y el test de normalidad y homocedasticidad de todas las variables medidas, empleando transformaciones numéricas. También se empleó el análisis de la varianza (ANOVA) de una y dos vías y el test a posteriori de Fisher para comprobar las diferencias entre las medias de las variables de los distintos tratamientos (tipo de suelo, tipo de fertilizante y concentraciones de $\mathrm{P}$ añadido). En todos los análisis estadísticos se empleó el programa IBM SPSS Statistics 22.

Finalmente, el estudio unifactorial con el tratamiento estadístico de la diferencia entre tratamientos (factores de variación: Tipo de Suelo, Tipo de fertilizante, Concentración de $\mathrm{P}$ añadido) para la determinación de la concentración óptima de la estruvita como fuente de fósforo en Lepidium sativum se dividió en los siguientes apartados: porcentaje de germinación, porcentaje de longitud de la raíz, porcentaje de la longitud del tallo y porcentaje del índice de germinación.

\section{RESULTADOS Y DISCUSIÓN}

A continuación, se reportan los resultados obtenidos de los ensayos realizados para la determinación de la concentración óptima de la estruvita como fuente de P en Lepidium sativum.

\section{Porcentaje de germinación (PG)}

No existe diferencia significativa en el porcentaje de germinación de semillas $L$. sativum que pudieran ser afectadas por el tipo de suelo. Los datos obtenidos están dentro de $96 \%$ en suelos silíceos sobre granodiorita y $94 \%$ en suelos carbonatados sobre marga. Tampoco se encontró diferencia significativa en el tipo de fertilizante aplicado que infiera negativamente en la germinación, teniendo en los dos casos, estruvita y suelo ácido el $95 \%$ de germinación (Tabla 3). En base a la terminología empleada y los datos de Kameswara et al. (2007), valores superiores del $80 \%$ de germinación de semillas testadas confieren la buena calidad de las semillas empleadas en el proceso. En tanto, las concentraciones añadidas desde 0 a $32 \mathrm{ppm}$ de $\mathrm{P}$, las interacciones tipo de suelo-tipo de fertilizante, tipo de suelo-concentración y tipo de fertilizante-concentración no muestran diferencias significativas en el proceso de germinación de semillas.

\begin{tabular}{|c|c|c|c|}
\hline $\begin{array}{l}\text { Factor / } \\
\text { Parámetro }\end{array}$ & $\begin{array}{l}\text { Fertilizante }^{a} \\
\text { estruvita/SS }\end{array}$ & $\begin{array}{c}\text { Suelo }^{\mathbf{a}} \\
\text { granodiorita/ } \\
\text { marga } \\
\end{array}$ & $\begin{array}{c}\text { Concentración } \mathrm{P} \text { en } \\
\text { solución }\left(\mu \mathrm{gL}^{-1}\right)^{\mathrm{b}} \\
0 / 3 / 6 / 10 / 17 / 32\end{array}$ \\
\hline G $(\%)^{b}$ & $95 / 95$ & $96 / 94$ & b97 / 94 / 97 / 95 / 94 / 95 \\
\hline LR (\%) & $72 / 59^{* * *}$ & $68 / 57^{* *}$ & b70 / 68 / 70 / 64 / 64 / 61 \\
\hline LT $(\%)^{c}$ & $122 / 74^{* * *}$ & $99 / 94$ & ${ }^{c} 100 / 99 / 95 / 102 / 97 / 97$ \\
\hline IG (\%) & $69 / 56^{* *}$ & $66 / 54^{* *}$ & ${ }^{\mathrm{b}} 68 / 65 / 68 / 61 / 61 / 58$ \\
\hline
\end{tabular}

Tabla 3. Medias de los parámetros de desarrollo de Lepidium sativum según factores tipo de fertilizante, tipo de suelo y la concentración de $P$ añadido. Table 3. Growth parameter means of Lepidium Sativum according to fertilizer type, soil type and the concentration of added P.

aSignificancia del test de Student para dos grupos: ${ }^{*}<0.05,{ }^{* *}<0.01,{ }^{* * *}<$
0.001 .

b: Diferentes letras muestran diferencias significativas (test de Stuart-Newman-Keuls, $p<0.05$ ).

$c$ : Diferentes letras implican diferencias significativas (Test de U de MannWhitney, $p<0.05)$.

G: Germinación; LR: Longitud de la Raíz; LT: Longitud del Tallo; IG: Índice de Germinación; SS: superfosfato simple; P: fósforo.

aSignificant of Student test for two groups: ${ }^{*}<0.05,{ }^{* *}<0.01,{ }^{* * *}<0.001$.

b: Different letters show significant differences (Stuart-Newman-Keuls test, $p<0.05$ ).

c: Different letters show significant differences ( $\mathrm{U}$ de Mann-Whitney test, $p$ $<0.05)$.

G: Germination; LR: Root Length; LT: Stem Length; IG: Germination Index; SS: Superphosphate simple; P: Phosphor.

\section{Porcentaje de longitud de la raíz (PLR)}

La longitud de la raíz en los ensayos difiere significativamente en los dos tipos de suelos empleados $(p<0.01)$, teniendo porcentajes bajos en suelos básicos en comparación con el suelo ácido (57 y $68 \%$, respectivamente). Este efecto se repite cuando se aplica el fertilizante $(p<0.001)$ con porcentajes bajos en superfosfato y ligeramente elevados en estruvita ( 59 y $72 \%$, respectivamente). De forma contraria, se observa que la adición de $\mathrm{P}$ de 0 a 32 ppm, sin importar su origen, no provoca diferencias significativas en la longitud de la raíz en los tres bloques de interacciones tipo de suelo-tipo de fertilizante-concentraciones (Tabla 3). El análisis general, muestra que la inhibición del desarrollo de la raíz se presenta mayoritariamente en suelos carbonatados y con superfosfato, este hecho puede confirmar el papel esencial que juega la presencia de ácidos orgánicos tanto en las plantas como en 
los suelos para la disolución de $\mathrm{P}$ de la estruvita y por ende su fácil adsorción en las plantas (Talboys et al., 2016). Las medias del test de Stuart-Newman-Keuls tienen similitud en el porcentaje de crecimiento radicular de las concentraciones en ppm de P 0 (70\%), 3 (68\%), 6 (70\%), 10 (64\%), 17 (64\%) y 32 (61\%), pero el mayor índice de crecimiento se observa en las concentraciones de 0 a 6 ppm de P.

\section{Porcentaje de la longitud del tallo (PLT)}

No se encontraron diferencias significativas en el porcentaje de la longitud del tallo en función del tipo de suelo, ( $99 \%$ suelo silíceo y $94 \%$ suelo carbonatado), pero si con el tipo de fertilizante aplicado $(p<0.001)$, teniendo mayores porcentajes de longitud de tallo en estruvita del $122 \%$ y en superfosfato del $74 \%$. Según el test de U de Mann-Whitney (Tabla 3), la longitud del tallo no muestra diferencias significativas en función de la concentración de $\mathrm{P}$ añadido (todos los niveles, de 0 a 32 ppm). Los mayores porcentajes de longitud de tallo, por tanto, están asociados con la adición de estruvita, mientras que los efectos reductores del crecimiento del tallo se presentan al agregar superfosfato como fuente de P. Este resultado explica de forma general que gran parte de la estruvita en contacto con suelos ácidos y la exudación misma de ácidos orgánicos de L. Sativum ayudan a incrementar la solubilidad de la estruvita y la fijación rápida de $\mathrm{P}$ en la planta, similares ensayos obtenidos en suelos ácidos ricos en arcilla (Plaza et al., 2007; Vogel et al., 2015; Talboys et al., 2016;), el incremento de la biomasa vegetal está en función del aumento de la concentración de $\mathrm{P}$ sin llegar a la saturación de Erel et al. (2008) y la funcionalidad de los ácidos orgánicos de las especies vegetales T. aestivum y F. esculentum en la solubilidad de P (Talboys et al., 2016). La ANOVA de dos vías de las tres combinaciones tipo de suelo-tipo de fertilizanteconcentración de $\mathrm{P}$, tampoco indica diferencias significativas en la longitud del tallo.

\section{Porcentaje del índice de germinación (PIG)}

El índice de germinación en el tipo de suelo tiende a presentar diferencias significativas $(p<0.01)$, en el orden de 64 y $54 \%$ para suelos ácidos y básicos, respectivamente. En el segundo caso, se cuenta con 69 y $56 \%$ de índice de germinación en estruvita y superfosfato $(p<0.01)$, resultados esperados y análogos a la longitud de la raíz en los rangos de inhibición de crecimiento de la raíz (Tabla 3). La ANOVA de dos vías en la combinación de interacciones de tipo suelotipo fertilizante-concentración de $\mathrm{P}$ agregado no son significativamente distintos. El estudio de las medias del índice de germinación muestra una disminución considerable cuando se incrementa la concentración de P en ppm: 0 y $6(68 \%), 3$ (65\%), 10 a 17 (61\%) y 32 (58\%). Los resultados indican que la dosis óptima de fertilizante (estruvita) para que la planta alcance su desarrollo óptimo, sin que esta resulte ser tóxica o como fuente inhibitoria para su desarrollo es de 0 a 6 ppm, misma que se corrobora con los estudios realizados por Erel et al. (2008), además la adición excesiva de fertilizante implica un consumo de $\mathrm{P}$ innecesario por parte de la planta
(Mustafa y Gary, 2014) debido a que la máxima absorción de $P$ en la planta se da en las primeras cosechas, proceso similar al ensayo realizado en cuanto a la etapa de desarrollo de la planta.

La planta modelo L. sativum utilizada en los bioensayos de fitoxicidad con medios de cultivo de los dos tipos de suelos (granodiorita y marga) y el fertilizante estruvita y superfosfato no mostraron efecto tóxico en la planta a concentraciones entre 0 a 6 ppm, alcanzando valores óptimos en la germinación de semillas y en la estimulación del crecimiento del tallo, dicho resultado se corrobora con la bibliografía reportada (Naveed et al., 2017). Los valores de los porcentajes de germinación de las semillas L. sativum obtenidos en los ensayos indicaron la buena calidad de las semillas seleccionadas, en función de los parámetros empleados de Kameswara et al. (2007). Se observaron valores del 95\% de germinación relativamente altos en suelos de granodiorita con estruvita que en marga y superfosfato. Los porcentajes del crecimiento de la raíz se diferencia entre un $10 \%$ del tipo de suelo y tipo de fertilizante, teniendo mayor crecimiento en suelos de pH 6.74 y estruvita (68 y 70\%), la explicación a estas diferencias se debe al factor concentración, que condiciona el crecimiento siendo las concentraciones de $P$ añadido de 0 a 6 ppm los que inducen el crecimiento de la raíz, mientras que por arriba de $6 \mathrm{ppm}$ inhiben el crecimiento radicular. Estos resultados favorecieron el crecimiento del tallo por arriba del $100 \%$ con mayor estimulación en suelos de granodiorita y estruvita (99 y 122\%), así mismo, en el análisis del índice de germinación se determina que la planta alcanza su desarrollo óptimo al disponer de una concentración de $\mathrm{P}$ adecuado, que en este caso se da a bajas concentraciones (64 y $54 \%$ suelos ácidos y básicos; 69 y $56 \%$ en estruvita y superfosfato). Porcentajes de índice de germinación de 60 a $70 \%$ en estruvita y TSP en suelos arenosos (Vaneeckhaute et al., 2016), valores de $150 \%$ en estudios en bioensayos con lechuga (El Fels et al., 2014), la estruvita de estiércol de pollo los PLT en $120 \%$ (Khan et al., 2014), el PIG de diferentes especies vegetales está dentro del 20 a 55\% según tratamiento de la estruvita (Mbarka et al., 2010) y 15 a 60\% uso de residuos (Ofosu-Budu et al., 2010); son similares a los que se obtuvieron en estruvita y superfosfato.

\section{CONCLUSIONES}

La disponibilidad de fósforo de la estruvita estará condicionada fundamentalmente por la textura, propiedades mineralógicas del suelo y en cierta forma, por la capacidad de las plantas para segregar ácidos orgánicos a fin de favorecer la solubilidad del fertilizante, así mismo, la adsorción de ésta en la planta. Los mayores efectos inhibitorios del desarrollo de la planta se presentaron en suelos carbonatados (longitud de raíz e índice de germinación) y resultados óptimos de desarrollo en suelos sobre granodiorita y con estruvita. El contenido de fósforo de la estruvita, en los dos tipos de suelos estudiados, demuestra la viabilidad de este material para su uso en la agricultura, encontrando que las condiciones óptimas de desarrollo de la planta se presentan 
a bajas concentraciones de estruvita y principalmente en suelos sobre granodiorita.

\section{REFERENCIAS}

Boluda, R., Roca-Pérez, L. y Marimón, L. 2011. Soil plate bioassay: An effective method to determine ecotoxicological risks. Chemosphere. 84: 1-8. In: https://doi.org/10.1016/j. chemosphere.2011.02.013

Cabeza, R., Steingrobe, B., Römer, W. y Claassen, N. 2011. Effectiveness of recycled $P$ products as $P$ fertilizers, as evaluated in pot experiments. Nutrient Cycling in Agroecosystems. 91: 173-184. In: https://doi.org/10.1007/ s10705-011-9454-0

El Fels, L., Zamama, M., El Asli, A. y Hafidi, M. 2014. Assessment of biotransformation of organic matter during co-composting of sewage sludge-lignocelullosic waste by chemical, FTIR analyses, and phytotoxicity tests. International Biodeterioration \& Biodegradation. 87: 128-137. In: https:// doi.org/10.1016/j.ibiod.2013.09.024

Erel, R., Dag, A., Ben-Gal, A., Schwartz, A. y Yermiyahu, U. 2008. Flowering and fruit set of olive trees in response to Nitrogen, Phosphorus, and Potassium. Journal of the American Society for Horticultural Science. 133(5): 639-647.

González-Poncer, R. y García-López, D. 2007. Evaluation of struvite as a fertilizer: a comparison with traditional $P$ sources. Agrochimica. 51: 30-308. In:

https://pubag.nal.usda.gov/pubag/downloadPDF. xhtml?id=28305\&content=PDF

Huijun, W., Yongliang, Z., Zengwei, Y. y Liangmin, G. 2016. A review of phosphorus management through the food system: identifying the roadmap to ecological agriculture. Journal of Cleaner Production. 114: 45-54. In: https://doi. org/10.1016/j.jclepro.2015.07.073

Johnston, A. y Richards, I. 2003. Effectiveness of different precipitated phosphates as phosphorus sources for plants. Soil Use Management. 19: 45-49. In: https://doi. org/10.1111/j.1475-2743.2003.tb00278.x

Kameswara, N., Hanson, J., Ehsan, M., Ghosh, K., Nowell, D. y Larinde, M. 2007. Manual of seed handling in genebanks. Handbooks for Genebanks No. 8. Bioversity International, Rome, Italy.

Kataki, S. y Baruah, D. 2018. Prospects and issues of phosphorus recovery as struvite from waste streams. Handbook of Environmental Materials Management. 1-50. In: https://doi. org/10.1007/978-3-319-58538-3_19-1

Kataki, S., West, H., Clarke, M. y Baruah, D. 2016. Phosphorus recovery as struvite from farm, municipal and industrial waste: Feedstock suitability, methods and pre-treatments. Waste Management. 49: 437-454. In: https://doi. org/10.1016/j.wasman.2016.01.003.

Khan, N., Clark, I., Sánchez-Monedero, M., Shea, S., Meier, S. y Bolan, N. 2014. Maturity indices in co-composting of chicken manure and sawdust with Biochar. Bioresource Technology. 168: 245-251. https://10.1016/j.biortech.2014.02.123

Maaß, O., Grundmann, P. y Bock un Polach, C. 2014. Added-value from innovative value chains by establishing nutrient cycles via struvite. Resources, Conservation and Recycling. 87: 126136. In: https://doi.org/10.1016/j.resconrec.2014.03.012
Massey, M., Davis, J., Ippolito, J. y Sheffield, R. 2009. Effectiveness of recovered magnesium phosphates as fertilizers in neutral and slightly alkaline soils. Agronomy Journal. 101(2): $323-$ 329.

Mbarka, G., Mongi, F.y Sami, S. 2010. Bioassay and use in irrigation of untreated and treated wastewaters from phosphate fertilizer industry. Ecotoxicology and Environmental Safety. 73: 932-938. In: https://doi.org/10.1016/j. ecoenv.2009.12.021

Metson, G., MacDonald, G., Haberman, D., Nesme, T. y Bennett, E. 2016. Feeding the Corn Belt: Opportunities for phosphorus recycling in U.S. agriculture. Science of the Total Environment. 542: 1117-1126. In: http://dx.doi. org/10.1016/j.scitotenv.2015.08.047

Mustafa, N. y Gary, M. 2014. The Freundlich adsorcion isotherm constants and prediction of phosphorus bioavailability as affected by different phosphorus sources in two Kansas soils. Chemosphere. 99: 72-80. In: https://doi.org/10.1016/j. chemosphere.2013.10.009

Naveed, A., Soomin, S., Seunggun, W. y Changsix, R. 2017. Struvite recovered from various types of wastewaters: characteristics, soil leaching behaviour, and plant growth. Land Degradation and Development. 29(9): 2864-2879. In: https://doi.org/10.1002/ldr.3010

Ofosu-Budu, G., Hogarh, J., Fobil, J., Quaye, A., Danso, S. y Carboo, D. 2010. Harmonizing procedures for the evaluation of compost maturity in two compost types in Ghana. Resources, Conservation and Recycling. 54: 205-209. In: https://doi.org/10.1016/j.resconrec.2009.08.001

Pastor, L., Mangin, D., Barat, R. y Seco, A. 2008. A pilot-scale study of struvite precipitation in a stirred tank reactor: conditions influencing the process. Bioresources Technology. 99: 62856291. In: https://doi.org/10.1016/j.biortech.2007.12.003

Plaza, C., Sanz, R., Clemente, C., Fernández, J., González, R., Polo, A. y Colmenarejo, M. 2007. Greenhouse evaluation of struvite and sludges from municipal wastewater treatment works as phosphorus sources for plants. Journal of Agricultural and Food Chemistry. 55: 8206-8212. In: https://doi.org/10.1021/ jf071563y

Talboys, P., Heppell, J., Roose, T., Healey, J., Jones, D. y Winthers, P. 2016. Struvite: a slow-release fertilizer for sustainable phosphorus management?. Plant and Soil. 401(1): 109-123. In: https://10.1007/s11104-015-2747-3

Vaneeckhaute, C., Janda, J., Vanrolleghem, P., Tack, F. y Meers, E. 2016. Phosphorus use efficiency of bio-based fertilizers: Bioavailability and fractionation. Pedosphere. 26(3): 310325. In: https://doi.org/10.1016/S1002-0160(15)60045-5

Vogel, T., Nelles, M. y Eichler-Löbermann, B. 2015. Phosphorus application with recycled products from municipal waste water to different crop species. Ecological Engineering. 83: 466-475. In: https://doi.org/10.17221/513/2017-PSE

Wang, Y., Zhang, T., Hu, Q. y Tan, C. 2016. Phosphorus source coefficient determination for quantifying phosphorus loss risk of various animal manures. Geoderma. 278: 23-31. In: https://doi.org/10.1016/j.geoderma.2016.05.008

Zucconi, F., Pera, A., Forte, M. y De Bertoli, M. 1981. Evaluating toxicity in immature compost. Biocycle. 22: 54-57. 